\title{
INHALED NITRIC OXIDE IS NOT A NEGATIVE INOTROPIC AGENT IN A PORCINE MODEL OF PULMONARY HYPERTENSION
}

Daniel J. Goldstein, $\mathrm{MD}^{\mathrm{a}}$

David A. Dean, MD ${ }^{a}$

Arthur Smerling, $\mathrm{MD}^{\mathrm{b}}$

Mehmet C. Oz, MD ${ }^{\mathrm{a}}$

Daniel Burkhoff, $\mathrm{MD}^{\mathrm{c}}$

Marc L. Dickstein, MD $^{\mathrm{b}}$
Background: Reports of pulmonary edema complicating inhaled nitric oxide therapy in patients with chronic heart failure and pulmonary hypertension have raised the concern that inhaled nitric oxide may have negative inotropic effects. Methods and results: We investigated the effect of multiple doses of inhaled nitric oxide $(20,40$ and $80 \mathrm{ppm})$ on left ventricular contractile state in 10 open-chest pigs. Pressure-volume loops were generated during transient preload reduction to determine the end-systolic pressure-volume relationship and the stroke work-end-diastolic volume relation. Inhaled nitric oxide had no effect on systemic vascular resistance, cardiac output, end-systolic pressure-volume relationship or stroke workend-diastolic volume relation under normal conditions. After induction of pulmonary hypertension (intravenous thromboxane $A_{2}$ analog), inhalation of nitric oxide $(80 \mathrm{ppm})$ resulted in a reduction in pulmonary vascular resistance (mean \pm standard error of the mean) from $10.4 \pm 3$ to $6.5 \pm 2$ Wood units $(p<0.001)$ and in pulmonary artery pressure from $44 \pm 4$ to $33 \pm 4 \mathrm{~mm} \mathrm{Hg}(p<0.05)$. Left ventricular end-diastolic volume rose from $53 \pm 9 \mathrm{ml}$ to $57 \pm 10 \mathrm{ml}(p=0.02)$. No statistically significant change in cardiac output or systemic vascular resistance was observed. Inhaled nitric oxide had no effect on end-systolic pressure-volume relationship or stroke work-end-diastolic volume relation. Conclusions: In a porcine model of pulmonary hypertension, inhaled nitric oxide does not impair left ventricular contractile function. Therefore the cause of pulmonary edema observed in some patients receiving inhaled nitric oxide is not due to a negative inotropic action of this therapy. (J Thorac Cardiovasc Surg 1997; 114:461-6)
Inhaled nitric oxide (NO) is currently being used in a number of clinical situations to selectively reduce pulmonary vascular resistance. ${ }^{1,2}$ When given by the inhaled route, NO reduces pulmonary vascular resistance while leaving systemic vascular resistance

From the Departments of Surgery, ${ }^{\mathrm{a}}$ Anesthesiology, ${ }^{\mathrm{b}}$ and Medicine, ${ }^{\mathrm{c}}$ College of Physicians and Surgeons, Columbia University, New York, N.Y.

Poster presentation at the Sixty-eighth Scientific Sessions, American Heart Association, Anaheim, Calif., 1995.

Supported by the Start-Up Grant, Department of Surgery, College of Physicians and Surgeons, Columbia University.

Received for publication June 18, 1996; revisions requested Sept. 19, 1996; revisions received Feb. 13, 1997; accepted for publication Feb. 21, 1997.

Address for reprints: Daniel J. Goldstein, MD, Department of Surgery, 622 West 168th St., PO Box 268, New York, NY 10032.

Copyright (C) 1997 by Mosby-Year Book, Inc.

$0022-5223 / 97 \$ 5.00+0 \quad \mathbf{1 2 / 1 / 8 1 3 8 9}$ unaltered. This selectivity is a result of the rapid sequestration and inactivation of NO by hemoglobin before NO reaches the systemic circulation. ${ }^{3-5}$ However, reports of significant increases in pulmonary artery wedge pressure in patients with heart failure receiving inhaled NO therapy ${ }^{6}$ have raised important questions about this therapy. Emerging evidence that endogenous NO exerts negative inotropic effects ${ }^{7-9}$ has led some investigators to wonder whether the increase in pulmonary artery wedge pressure is a result of negative inotropic actions of inhaled NO therapy. ${ }^{10,11}$ Because of the potential of inhaled NO to counteract the pulmonary vasoconstrictive effects of cardiopulmonary bypass, ${ }^{12,13}$ and to evaluate the reversibility of pulmonary hypertension in cardiac transplant candidates, an understanding of the mechanisms by which inhaled NO causes elevations in pulmonary artery wedge pressure in patients with heart failure is clinically important. 
We designed this study to determine whether inhaled NO alters the ventricular contractile state. To this purpose, we studied the effect of inhaled NO in a porcine model of pulmonary hypertension in which state-of-the-art measurements of left ventricular (LV) function were collected at various concentrations of inhaled NO.

\section{Methods}

Animals received humane care in compliance with the "Principles of Laboratory Animal Care" formulated by the Institute of Laboratory Animal Resources and the "Guide for the Care and Use of Laboratory Animals" prepared by the Institute of Laboratory Animal Resources and published by the National Institutes of Health (NIH Publication No. 86-23, revised 1985). In addition, this study also conforms with the position of the American Heart Association on Research Animal Use.

Experimental preparation. The protocol was carried out in conditioned Yorkshire pigs weighing 45 to $50 \mathrm{~kg}$. Animals were anesthetized with acepromazine $(0.5 \mathrm{mg} / \mathrm{kg}$ intramuscularly), ketamine ( $20 \mathrm{mg} / \mathrm{kg}$ intramuscularly), and atropine (1 to $2 \mathrm{mg}$ intramuscularly). The lungs were mechanically ventilated, and ventilatory parameters were adjusted to maintain normocarbia as evidenced by frequent blood gas analyses. Normothermia was maintained by the use of two warming blankets and a heating lamp when necessary. Anesthesia was maintained with isoflurane $(1.5 \%$ to $2.0 \%)$ in $30 \%$ oxygen. The femoral artery was catheterized to monitor systemic blood pressure (mean arterial pressure) and to obtain samples for blood gas analysis. Through a midline sternotomy and longitudinal pericardiotomy, the inferior vena cava, pulmonary artery (PA), and ascending aorta were isolated with snares. Three $5 \mathrm{~F}$ micromanometer-tipped catheters (Millar Instruments, Inc., Houston, Tex.) were placed in the left atrium, right atrium, and PA through purse-string sutures in the atrial appendages and PA for continuous pressure monitoring. A $16 \mathrm{~mm}$ perivascular flow probe (Transonic System, Inc., Ithaca, N.Y.) was placed around the main PA. This flow probe was connected to an ultrasonic flowmeter that had a continuous display of cardiac output. A 5F Millar micromanometer was placed in the right external carotid artery and advanced into the ascending aorta to monitor aortic pressure. A twelveelectrode $(1.0 \mathrm{~cm}$ interelectrode spacing) $6 \mathrm{~F}$ micromanometer-tipped conductance catheter (Sentron, Federal Way, Wash.) was inserted through the left common carotid artery, advanced over the aortic valve into the $\mathrm{LV}$, and positioned in the apex to measure LV volume and pressure. This catheter was connected to a signal conditioner (Leycom 5, Cardiodynamics, The Hague, The Netherlands), which provides the conductance signal used for assessing $L V$ volume. This device also measures blood resistivity $(\rho)$, which is necessary for estimation of volume. Blood resistivity was measured in each animal before the start of the experimental protocol and after changes in experimental conditions. Proper positioning of the conductance catheter was ensured by palpating the tip in the $\mathrm{LV}$ apex and using only segmental volume signals that corresponded to segments residing in the ventricular chamber for generation of the total volume signal. The total conductance signal is affected not only by the conductivity of the blood in the chamber in which the catheter lies, but also by conductive structures that surround the chamber. The contribution to the total conductance signal from these surrounding structures is termed parallel conductance. We determined parallel conductance by the hypertonic saline technique using $5 \mathrm{ml}$ of $5 \%$ saline solution rapidly injected into the PA. ${ }^{14}$ Parallel conductance was recalculated after any change in experimental conditions (i.e., induction of pulmonary hypertension, administration of different concentrations of $\mathrm{NO}$ ) and before each measurement.

NO administration. After endotracheal intubation, animals received a mixture of oxygen and nitrogen to achieve an inspired fraction of oxygen of $32 \%$ to $35 \%$. NO gas (800 ppm in nitrogen) (Airco; BOC Gases, Murray Hill, N.J.) was delivered at concentrations of 20,40 , and 80 ppm by titrating the amount of nitrogen downward and the level of NO upward so as to maintain a constant inspired oxygen fraction and total gas flow rate of $5 \mathrm{~L} / \mathrm{min}$. The inspired concentration of NO was measured by an NO analyzer (Drager, Chantilly, Va.). Exhaled gases were scavenged and discarded to the atmosphere.

Study protocol. In the first part of the protocol, animals were studied in four conditions: baseline condition and during three different doses $(20,40$, and $80 \mathrm{ppm})$ of inhaled NO. Next, pulmonary hypertension was induced by continuous intravenous administration of a thromboxane $A_{2}$ analog $(9,11$-dideoxy- $11 \alpha, 9 \alpha$-epoxymethanoprostaglandin $\mathbf{F}_{2}$; Sigma Chemical Co., St. Louis, Mo.) at a rate that resulted in a mean PA pressure of greater than $35 \mathrm{~mm} \mathrm{Hg}(0.07$ to $0.15 \mu \mathrm{g} / \mathrm{kg}$ per minute). Animals were then restudied at the same four conditions just described. Data collected at each condition consisted of standard hemodynamic measurements as well as a series of LV pressure-volume loops measured before and during vena caval occlusion. The vena caval occlusion was limited to 10 seconds in duration to avoid reflex responses and was repeated if arrhythmias occurred. Ventilation was held during the period of data collection. Measurements were made 5 minutes after reaching a steady state NO concentration.

Data analysis. All analog signals were digitized at 200 $\mathrm{Hz}$ with the use of a 12-bit analog-to-digital board $(\mathrm{AD}$ Instruments, Milford, Mass.) and stored on a removable hard drive for subsequent analysis with IGOR analysis software (Wavemetrics Inc., Lake Oswego, Ore.). Pressures, flow, and volume data were subjected to a five-pass binomial smooth filter. End-diastolic pressure (EDP) was identified as the point just preceding the upstroke of the LV pressure wave. End-diastolic volume (EDV) was determined by the lower right hand corner of the pressure-volume loop. ${ }^{15}$

Two relations that describe ventricular contractile state were examined during vena caval occlusion; at least 7 cardiac cycles free of arrhythmias were used for each analysis:

End-systolic pressure-volume relationship (ESPVR). The slope and volume axis intercept of the end-systolic pressure-volume relationship (ESPVR) were determined as in 
Table I. Hemodynamics and LV contractile relations before induction of pulmonary hypertension $(n=10)$

\begin{tabular}{lccccc}
\hline Parameter & Control & NO 20 ppm & NO 40 ppm & NO 80 ppm & $p$ Value \\
\hline Hemodynamics & & & & & \\
CO & $5.7 \pm 0.3[4.9-6.5]$ & $5.6 \pm 0.7[4-7.2]$ & $5.5 \pm 0.6[4.1-6.9]$ & $5.3 \pm 0.4[4.4-6.2]$ & $>0.2$ \\
MAP & $68 \pm 3[62-74]$ & $68 \pm 2[62-73]$ & $67 \pm 3[61-73]$ & $67 \pm 3[62-73]$ & $>0.2$ \\
SVR & $11 \pm 1[7-15]$ & $12 \pm 2[7-17]$ & $12 \pm 2[7-17]$ & $12 \pm 2[8-16]$ & $>0.2$ \\
LVEDP & $7 \pm 2[3-11]$ & $8 \pm 1[5-10]$ & $8 \pm 1[5-11]$ & $8 \pm 1[5-11]$ & $>0.2$ \\
LVEDV & $57 \pm 4[48-66]$ & $56 \pm 4[47-65]$ & $57 \pm 4[47-67]$ & $56 \pm 4[46-66]$ & $>0.2$ \\
PAP & $23 \pm 1.8[19-27]$ & $18 \pm 1[16-21]$ & $19 \pm 1[16-22]$ & $19 \pm 1[16-22]$ & 0.043 \\
PVR & $2.8 \pm 0.4[1.9-3.7]$ & $2.1 \pm 0.3[1.3-2.9]$ & $2.2 \pm 0.4[1.3-3.2]$ & $2.3 \pm 0.4[1.3-3.2]$ & $>0.2$ \\
Contractility & & & & & $>0.2$ \\
ESPVR & $2.6 \pm 0.5$ & $2.9 \pm 0.5$ & $2.6 \pm 0.6$ & $-1 \pm 0.6$ & $>0.2$ \\
Mo & $-1 \pm 9$ & $5 \pm 8$ & $-1 \pm 9$ & $58 \pm 6$ & \\
SWEDV & $61 \pm 6$ & $57 \pm 6$ & $56 \pm 6$ & $26 \pm 8$ & \\
Vo & $27 \pm 8$ & $26 \pm 7$ & $26 \pm 8$ & & $>0.2$ \\
\hline
\end{tabular}

Figures are expressed as mean \pm standard error of the mean. Brackets indicate $95 \%$ confidence intervals. $C O$, Cardiac output $(\mathrm{L} / \mathrm{min}) ; M A P$, mean arterial pressure (mm Hg); SVR, systemic vascular resistance (Wood units); $L V E D P$, left ventricular end-diastolic pressure (mm $\mathrm{Hg}$ ); $L V E D V$, left ventricular end-diastolic volume; $P A P$, pulmonary artery pressure ( $\mathrm{mm} \mathrm{Hg}$ ); $P V R$, pulmonary vascular resistance; ESPVR, end-systolic pressure-volume relation (mm $\mathrm{Hg} / \mathrm{ml}) ; M o$, x-axis intercept of ESPVR line (ml); SWEDV, stroke-work end-diastolic volume $\left(\mathrm{erg} \cdot \mathrm{cm}^{-3} \cdot 10^{3}\right) ; V o$, X-axis intercept of SWEDV line (ml).

previous studies. ${ }^{15}$ In brief, the volume axis intercept (Vo) was initially assumed to be $0 \mathrm{ml}$ and values for end-systolic pressure and end-systolic volume were determined for each cardiac cycle by identifying the point at which the value of $P(t) /(V[t]-V o)$ reached a maximum. Regression analysis of these end-systolic pressure and end-systolic volume points provided an improved estimate of volume axis intercept. This updated volume axis intercept value was then substituted into the equation, and new values for end-systolic pressure and volume were obtained. Subsequent iterations were performed until the value of volume axis intercept changed by less than $2 \mathrm{ml}$.

Stroke work-end-diastolic volume relationship $(S W E D V)$. Stroke work was determined for each cardiac cycle by calculating the area within the respective pressure-volume loop. Stroke work-end-diastolic volume (SWEDV) was defined by the relationship between stroke work and end-diastolic volume. ${ }^{16}$

Cardiac output was determined by the integral of the PA flow waveform, and pulmonary and systemic vascular resistances were calculated by means of standard formulas.

Statistical analysis. Statistical analyses were performed with InStat software package for Macintosh on an Apple computer (Apple Computer, Cupertino, Calif.). Each relation (ESPVR, SWEDV) was described by a slope and a volume axis intercept obtained from linear regression analysis. The effect of NO on these two variables was assessed by analysis of variance. The impact of NO dose on standard hemodynamic parameters was also assessed by analysis of variance for repeated measures. The Bonferroni correction was applied to account for the fact that comparisons were made between multiple groups. A two-tailed $p$ value $<0.05$ was considered statistically significant.

\section{Results}

Hemodynamic data measured at baseline and at each NO dose $(n=10)$ before the induction of pulmonary hypertension are presented in Table I.
No change was observed in cardiac output, systemic vascular resistance, mean arterial pressure, or left ventricular end-diastolic pressure (LVEDP) or volume (LVEDV). Inhaled NO (all three doses) caused a slight reduction in PA pressure (mean \pm standard error of the mean) $(23 \pm 1.8 \mathrm{~mm} \mathrm{Hg}$ [19 to $27 \mathrm{~mm} \mathrm{Hg}, 95 \% \mathrm{CI}^{*}$ ] to $19 \pm 1 \mathrm{~mm} \mathrm{Hg}$ [16 to $22 \mathrm{~mm}$ $\mathrm{Hg}, 95 \% \mathrm{CI}] ; p=0.04$ ). Representative pressurevolume loops obtained during transient vena caval occlusion at baseline and during NO administration are shown in Fig. 1. At each dose of NO, the pressure-volume loops are superimposable with those measured at baseline (left panel). The contractile relations (ESPVR and SWEDV) derived from these loops were similarly unchanged by the administration of NO (Table I and Fig. 1, right panel).

Administration of thromboxane $\mathrm{A}_{2}$ analog resulted in an unstable preparation resulting from arrhythmias, severe systemic hypotension (mean arterial pressure $<40 \mathrm{~mm} \mathrm{Hg}$ ), or death in four animals; these animals were excluded from further study. In the remaining six animals, administration of thromboxane resulted in an elevation (mean \pm standard error of the mean) of baseline pulmonary vascular resistance from $2.8 \pm 0.4$ Wood units (1.9 to 3.5 Wood units, $95 \% \mathrm{CI}$ ) to $10.4 \pm 3$ Wood units (2.9 to 17.8 Wood units, 95\% CI) $(p=0.037)$, elevation of mean PA pressure from $23 \pm 2 \mathrm{~mm} \mathrm{Hg}$ (20 to $27 \mathrm{~mm} \mathrm{Hg}, 95 \% \mathrm{CI}$ ) to $43 \pm 4 \mathrm{~mm} \mathrm{Hg}$ (35 to $53 \mathrm{~mm} \mathrm{Hg}, 95 \% \mathrm{CI})(p=0.0011)$, and a reduction in cardiac output from $5.8 \pm 0.5 \mathrm{~L} / \mathrm{min}(2.2$ to 6.7 , $95 \% \mathrm{CI})$ to $4.5 \pm 0.9 \mathrm{~L} / \mathrm{min}(4.4$ to $7.1,95 \% \mathrm{CI})(p=$

${ }^{*} \mathrm{CI}=$ Confidence interval. 


\section{ESPVR}

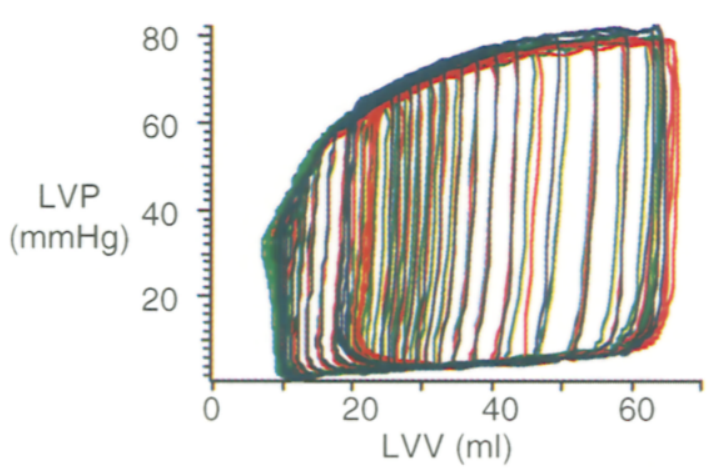

\section{PRSW}

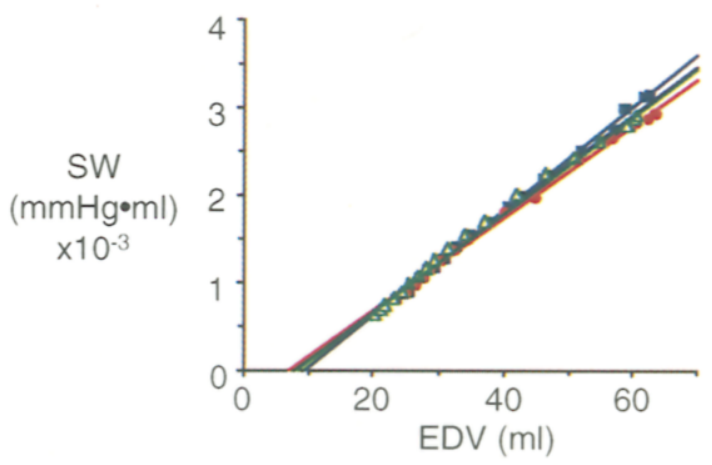

\begin{tabular}{|lll|}
\hline Baseline $\quad$ NO $20 \mathrm{ppm}$ & NO $40 \mathrm{ppm} \quad$ NO $80 \mathrm{ppm}$ \\
\hline
\end{tabular}

Fig. 1. Representative pressure-volume loops obtained during transient vena caval occlusion at baseline and during inhaled NO administration are shown. At each dose of NO $(20,40,80 \mathrm{ppm})$, the pressure volume loops are superimposable with those measured at baseline (left panel). The contractile relations (ESPVR and SWEDV) derived from these loops were similarly unchanged by the administration of NO (right panel). ESPVR, End-systolic pressure-volume relation; PRSW, preload recruitable stroke work; LVP, left ventricular pressure; $L V V$, left ventricular volume; $S W$, stroke work; $E D V$, end-diastolic volume.

Table II. Hemodynamics and $L V$ standard error of the mean contractile relations after induction of pulmonary hypertension $(n=6)$

\begin{tabular}{|c|c|c|c|c|c|}
\hline Parameter & $\begin{array}{l}\text { Pulmonary } \\
\text { hypertension }\end{array}$ & NO $20 \mathrm{ppm}$ & NO $40 \mathrm{ppm}$ & NO $80 \mathrm{ppm}$ & $p$ Value \\
\hline \multicolumn{6}{|c|}{ Hemodynamics } \\
\hline $\mathrm{CO}$ & $4.6 \pm 1[1.6-7.5]$ & $4.9 \pm 1[2.1-7.8]$ & $5.1 \pm 1[2.3-7.9]$ & $5.0 \pm 1[2-7.9]$ & $>0.2$ \\
\hline MAP & $72 \pm 7[52-92]$ & $80 \pm 6[64-97]$ & $78 \pm 5[65-92]$ & $79 \pm 6[62-96]$ & $>0.2$ \\
\hline SVR & $20 \pm 6[3-37]$ & $19 \pm 6[3-34]$ & $17 \pm 4[5-29]$ & $18 \pm 5[5-31]$ & 0.15 \\
\hline LVEDP & $8 \pm 2[3-13]$ & $10 \pm 2[5-15]$ & $11 \pm 2[6-16]$ & $10 \pm 2[4-17]$ & 0.18 \\
\hline LVEDV & $53 \pm 9[27-79]$ & $54 \pm 10[26-82]$ & $57 \pm 11[27-88]$ & $57 \pm 10[30-84]$ & 0.02 \\
\hline PAP & $44 \pm 4[35-53]$ & $35 \pm 4[24-45]$ & $33 \pm 4[22-44]$ & $33 \pm 4[21-44]$ & $<0.0001$ \\
\hline PVR & $10.4 \pm 3[2.9-18]$ & $7.5 \pm 3[0.2-15]$ & $6.3 \pm 2[0.9-12]$ & $6.5 \pm 2[1-12]$ & 0.0008 \\
\hline \multicolumn{6}{|l|}{ Contractility } \\
\hline ESPVR & $1.8 \pm 0.3$ & $2.2 \pm 0.7$ & $1.9 \pm 0.5$ & $2.1 \pm 0.5$ & $>0.2$ \\
\hline Mo & $-6 \pm 16$ & $-16 \pm 20$ & $-10 \pm 16$ & $-16 \pm 20$ & $>0.2$ \\
\hline SWEDV & $42 \pm 8$ & $48 \pm 4$ & $49 \pm 4$ & $49 \pm 5$ & $>0.2$ \\
\hline Vo & $24 \pm 13$ & $28 \pm 13$ & $29 \pm 11$ & $29 \pm 12$ & $>0.2$ \\
\hline
\end{tabular}

Figures are expressed as mean \pm standard error of the mean. Brackets indicate $95 \%$ confidence intervals. $C O$, Cardiac output (L/min); $M A P$, mean arterial pressure $(\mathrm{mm} \mathrm{Hg}) ; S V R$, systemic vascular resistance (Wood units); $L V E D P$, left ventricular end-diastolic pressure (mm Hg); $L V E D V$, left ventricular end-diastolid volume; $P A P$, pulmonary artery pressure $(\mathrm{mm} \mathrm{Hg}) ; P V R$, pulmonary vascular resistance; $E S P V R$, end-systolic pressure-volume relation (mm $\mathrm{Hg} / \mathrm{ml}) ; M o$, x-axis intercept of ESPVR line (ml); SWEDV, stroke-work end-diastolic volume (erg.cm-3.103) (erg $\left.\cdot \mathrm{cm}^{-3} \cdot 10^{3}\right) ; \mathrm{Vo}$, x-axis intercept of SWEDV line (ml).

$0.06)$. In this group, inhaled NO caused a reduction in PA pressure $(p<0.0001)$ and a reduction of pulmonary vascular resistance $(p=0.0008)$ at all three doses. An elevation in LVEDV at all three doses of inhaled NO was noted $(p=0.02)$, whereas no change in LVEDP was observed. A trend toward increased cardiac output was noted $(p=0.18$ )
(Table II). ESPVR and SWEDV again were unaffected by administration of inhaled NO at any dose. Results are summarized in Table II. Comparison of ventricular contractility (mean \pm standard error of the mean) before and after induction of pulmonary hypertension was ESPVR $2.03 \pm 0.73$ (1.25 to 2.8 , $95 \% \mathrm{CI})$ versus $1.81 \pm 0.32(0.39$ to $4.66,95 \% \mathrm{CI})$, 
$p=0.39$, and SWEDV $56.7 \pm 6(41.1$ to $71.2,95 \%$

CI) versus $47.4 \pm 6(31.8$ to $62.9,95 \% \mathrm{CI}), p=0.2$.

\section{Discussion}

The results of this study demonstrate that inhaled NO does not lead to alterations in LV contractile state. In fact, the only observed hemodynamic alterations associated with inhaled NO were reductions in PA pressure and pulmonary vascular resistance and a mild elevation in LVEDV. These findings are consistent with other reports of the hemodynamic effects of inhaled NO and further clarify the mechanism by which elevations in pulmonary capillary wedge pressure occur during this therapy.

Inhaled NO is currently an important therapy in a number of clinical situations for both diagnostic and therapeutic purposes. Inhaled NO is being used to test vasoreactivity in patients with heart failure during evaluation for cardiac transplantation ${ }^{17}$; fixed pulmonary hypertension is considered a contraindication for transplantation because of the prevalence of perioperative right heart failure ${ }^{18,19}$ Inhaled NO is also being used with increasing frequency in the perioperative management of right heart failure in patients undergoing heart transplantation or LV assist device implantation. ${ }^{20}$

Several reports of acute elevation in pulmonary artery wedge pressure in the heart failure populations have been published, ${ }^{6,10,11}$ and concern has emerged that the increase in LV filling pressure reflects negative inotropic actions of this therapy. This concern has been bolstered by laboratory reports of negative inotropic actions of NO in isolated myocyte preparations. ${ }^{7-9}$ However, NO is rapidly sequestered by hemoglobin in the blood and thereby should be inactivated long before reaching the systemic circulation. ${ }^{21}$ This rapid sequestration is supported by the lack of systemic vasodilatory effects in the setting of significant pulmonary vasodilation. It is therefore unlikely that when administered via the inhaled route, NO could exert any direct myocardial effects. $^{22,23}$ In fact, results of the present study failed to show any effects of inhaled NO on LV contractile function.

The mechanism by which inhaled NO leads to elevations in LV filling pressure remains to be answered. We observed an increase in LVEDV in response to pulmonary vasodilation and showed that this increase is not due to alterations in ventricular function. This finding is consistent with a recent theoretic analysis of the direct impact of pulmonary vasodilation on pulmonary venous pressure. ${ }^{24}$ In this study, Dickstein and Burkhoff ${ }^{24}$ showed that progressive pulmonary vasodilation is associated with increases in pulmonary wedge pressure independent of changes in ventricular function. Importantly, it was also shown that the magnitude of increase in pulmonary wedge pressure was most affected by intravascular volume. The present study was conducted under euvolemic conditions, and we still observed increases in LVEDV. Operating on the flat portion of the normal diastolic compliance curve, it is not surprising that we observed no significant increase in LVEDP. It is likely that with larger intravascular volumes and/or operating on the steeper portion of a diastolic compliance curve, the increases in LVEDP relative to LVEDV would be more profound.

In summary, we have demonstrated that inhaled NO therapy does not affect the inotropic state of the normal myocardium. Under conditions of pulmonary hypertension and an intact $L V$, inhaled NO therapy produces selective pulmonary vasodilation and does not affect myocardial contractile performance. The rise in left atrial pressure in patients with preexisting $L V$ dysfunction receiving inhaled NO therapy appears to be a direct consequence of pulmonary vasodilation.

\section{REFERENCES}

1. Rich G, Murphy G, Roos C, Johns R. Inhaled nitric oxide: selective pulmonary vasodilatation in cardiac surgical patients. Anesthesiology 1993;78:1028-35.

2. Pepke-Zaba J, Higenbottam TW, Dinh Xuan AT, Stone D, Wallwork J. Inhaled nitric oxide as a cause of selective pulmonary vasodilation in pulmonary hypertension. Lancet 1991;338:1173-4.

3. Edwards AD. The pharmacology of inhaled nitric oxide. Arch Dis Child 1995;72:F127-30.

4. Moncada S. The 1991 UIf von Euler Lecture. The L-arginine: nitric oxide pathway. Acta Physiol Scand 1992;145:201-7.

5. Moncada S, Palmer RMJ, Higgs EA. Nitric oxide: physiology, pathophysiology and pharmacology. Pharmacol Rev 1991;43: $109-42$.

6. Bocchi EA, Bacal F, Auler JOC, Carmone MJC, Bellotti G, Pileggi F. Inhaled nitric oxide leading to pulmonary edema in stable severe heart failure. Am J Cardiol 1994;74:70-1.

7. Brady AJ, Warren JB, Poole-Wilson PA, Williams TJ, Harding SE. Nitric oxide attenuates cardiac myocyte contraction. Am J Physiol 1993;265:H176-82.

8. Balligand JL, Ungureanu D, Kelly RA, Kobsik L, Pimental $\mathrm{D}$, Michel $\mathrm{T}$, et al. Abnormal contractile function due to induction of nitric oxide synthesis in rat cardiac myocytes follows exposure to activated macrophage conditioned medium. J Clin Invest 1993;91:2314-9.

9. Finkel MS, Oddis CV, Jacob TD, Watkins SC, Harrier BG, Simmons RL. Negative inotropic effects of cytokines on the heart mediated by nitric oxide. Science 1992;257:387-9.

10. Loh E, Stamler JS, Hare JM, Loscalzo J, Colucci WS. 
Cardiovascular effects of inhaled nitric oxide in patients with left ventricular dysfunction. Circulation 1994;90:2780-5.

11. Semigran MJ, Cockrill BA, Kacmarek R, Thompson BT, Zapol WM, Dec GW, et al. Hemodynamic effects of inhaled nitric oxide in heart failure. J Am Coll Cardiol 1994;24:982-8.

12. Beghetti M, Habre W, Friedli B, Berner M. Continuous low dose inhaled nitric oxide for treatment of severe pulmonary hypertension after cardiac surgery in pediatric patients. $\mathrm{Br}$ Heart J 1995;73:65-8.

13. Wessel DL, Adatia I, Giglia TM, Thompson JE, Kulik TJ. Use of inhaled nitric oxide and acetylcholine in the evaluation of pulmonary hypertension and endothelial function after cardiopulmonary bypass. Circulation 1993;88:2128-38.

14. Burkhoff D, van der Velde E, Kass D, Baan J, Maughan WL, Sagawa K. Accuracy of volume measurement by conductance catheter in isolated ejecting canine hearts. Circulation 1985; 72:440-7.

15. Sagawa K, Suga H, Shoukas AA, Bakalar KM. End-systolic pressure/volume ratio: a new index of ventricular contractility. Am J Cardiol 1977; 40:748-53.

16. Glower DD, Spratt JA, Snow ND, Kabas JS, Davis JW, Olsen $\mathrm{CO}$, et al. Linearity of the Frank-Starling relationship in the intact heart: the concept of preload recruitable stroke work. Circulation 1985;71:994-1009.

17. Addonizio LJ, Gersony WM, Robbins RC, et al. Elevated pulmonary vascular resistance and cardiac transplantation. Circulation 1987;76(Suppl):V52-5.

18. Kirklin JK, Naftel DC, Kirklin JW, Blackstone EH, WhiteWilliams C, Bourge RC. Pulmonary vascular resistance and the risk of heart transplantation. J Heart Transplant 1988;7: 331-6.

19. Kieler-Jensen N, Ricksten SE, Stenqvist O, Bergh $\mathrm{CH}$, Lindelov B, Wennmalm A, et al. Inhaled nitric oxide in the evaluation of heart transplant candidates with elevated pulmonary vascular resistance. J Heart Lung Transplant 1994; 13:366-75.

20. Salmonsen RF, Kaye D, Esmorre DS. Inhalation of nitric oxide provides selective pulmonary vasodilation aiding mechanical cardiac assist with Thoratec left ventricular assist device. Anaesth Intens Care 1994;22:209-10.

21. Rimar S, Gillis CN. Selective pulmonary vasodilation by inhaled nitric oxide is due to hemoglobin inactivation. Circulation 1993;88:2884-7.

22. Pearl RG. Inhaled nitric oxide: the past, the present and the future. Anesthesiology 1993;78:413-16.

23. Billiar TR. Nitric oxide: novel biology with clinical relevance. Ann Surg 1995;221:339-49.

24. Dickstein ML, Burkhoff $D$. The effect of nitric oxide administration on pulmonary venous pressure. J Heart Lung Transplant 1996;15:715-21. 